\title{
Research and application on Optimal Path Analysis in mining roadway ZHANG Zhihua $^{1,2, a}$, TIAN Kunrui ${ }^{1,2, b}$, YANG Shuwen ${ }^{1,2, c}$, and YANG Jun 1,2,d Yan Haowen ${ }^{1,2, e}$ \\ ${ }^{1}$ faculty of geomatics, Lanzhou Jiaotong University, Lanzhou China, \\ ${ }^{2}$ Gansu Provincial Engineering Laboratory for National Geographic State Monitoring, Lanzhou, China, \\ azhzhihua99@163.com, btiankunrui@126.com, '825198827@qq.com, d123072613@qq.com, \\ e haowen2010@gmail.com
}

Keywords: Optimal path analysis; Roadway Networks; Algorithms.

\begin{abstract}
It is an urgent problem about how to apply the GIS's powerful spatial analysis function to mine. On the basis of analysis on network elements and path analysis algorithm, the paper presented three models of optimal path analysis and the concrete algorithm steps. Under Windows XP, the optimal path methods were realized by using $\mathrm{VC}++$ and it was verified by applying specific experimental data.
\end{abstract}

\section{Introduction}

With development of geographic information systems and computer science, the digital mine construction has been greatly promoted. Theory and methods of geographic information system applying to mine production, not only improved the productivity of the mine, but also provided the necessary technical support for mine safety. Tunnels of mine have three-dimensional network characteristics. Therefore, to establish a mine geographic network space is an important task of digital mine, which is also the basis of mine disaster prevention research, major accident processing, decision management and other related analysis [1]. This article started with the characteristics of the roadway network, firstly to build the roadway network, and research on optimal path analysis, in order to provide the necessary technical support for the mine rescue.

\section{Building roadway network}

The basic elements of the mine roadway network have nodes, arcs, site, weight, resources, corners, etc. Node which is the most basic elements in roadway network analysis is the most basic elements in roadway network analysis; arcs correspond to a variety of linear elements in the network. In mine production, a various of roadways, wires, tub track which can be abstracted into arcs, only the centerlines of roadways are considered as the arcs of the network in order to reflect the distribution in space of the roadway. In the roadway network, the site is the node position for loading or unloading ore; weights used to express the costs which is required through an arc or node, expressed in many different ways, may be static or dynamic, in the roadway network, it can be the length of roadways, or may also be the time passed through the roadways. With these elements of the network, based on graph theory, we can build the graph of roadway network. $G=<N, E, W>$, where $N(v)$ is on behalf of the node set, $E(i, j)$ indicates arcs connecting node $i$ and $j, W(i, j)$ represents the weight of the arc.

\section{Path analysis algorithm}

Since the shortest path analysis and optimal path analysis use roughly the same algorithm, so we study and analyze the status of the shortest path algorithm. 
Many scholars at home and abroad have conducted research on path analysis, the most classic method of shortest path algorithm is proposed by EW Dijkstra in 1959 which generated the shortest path according to path length in ascending order.

Dijkstra algorithm adapting greedy strategy with strong anti-poor nature, which is widely used, has become preferred algorithm on the development platform for GIS network analysis module at home and abroad.Dijkstra algorithm is the most complete algorithm in current theory as known[3], Advantage of this algorithm: program design is simple and versatile; Disadvantages: it is not for two particular points, therefore less efficient; adjacency matrix occupies with enormous space induce to a serious waste of computer resources. There are many improved algorithm about Dijkstra algorithm, for example, using a binary heap priority queue achieve Dikstra algorithm [4,5], Geostar adopts quick sort of FIFO (first-in, first-out queue, First Input First Output, FIFO) queue to achieve Dijkstra algorithm [6].The paper[7] realized Dijkstra shortest path algorithm based on quad heap priority queue and inverse adjacency list. This method not only greatly improved the efficiency and flexibility of the algorithm, but also was applied to the vehicle navigation system under technical support of GPS.

At the random pair points of the shortest path analysis, Floyd proposed algorithm in 1962, which used to calculate the network shortest path between each pair of vertices, namely Floyd algorithm, which can solve the negative value of weight. Under the worst case, run-time complexity of Floyd algorithm is $\mathrm{O}\left(\mathrm{n}^{3}\right)$, Dijkstra algorithm execute $\mathrm{n}$ times(take different vertices), the same goal can be achieved for solving the shortest path between all nodes. As to dense graph, the running time complexity of Dijkstra algorithm is $\mathrm{O}\left(\mathrm{n}^{3} \log n\right)$. In contrast, Floyd algorithm is better. Matrix algorithm is mainly used to calculate the shortest path of each pair vertices in the network, and in the meanwhile it can find the secondary shortest path, whose running time complexity is $\mathrm{O}\left(\mathrm{n}^{4}\right)$.

The optimal path is that the path possesses the least impedance between two nodes within the network. The optimal path includes many meanings, not only points to the shortest distance with general geographic sense, but also refers to the shortest time, least-cost, the highest line utilization etc. from the perspective of the network model, solving of optimal path between two nodes is to find an least resistance strength through the path. The cases to choose optimal path include: (A) the shortest distance; (B) the lease time or cost; (C) try to take the main road firstly. The shortest path optimization is a static optimization, regardless of the real-time traffic conditions, only associated with the structure of transport network. Least time optimization is a dynamic optimization, and real-time traffic conditions are closely related. Try to take the main road firstly is another optimization at (A) or (B) added as constraint under the premise.

\section{optimal path analysis on Roadway network}

Two source optimal path analysis. Two sources optimal path analysis refers to that start from both different source nodes to the termination nodes, which this process take a minimum cost required [12]. Two source optimal path analysis exists many forms, for example, starting from two different source nodes and arriving to a same termination node, that is two-sources-one-destination (TSOD), as Fig. 1 is shown, there is a local roadway in network diagram, node 1and 3 are source nodes, node 2 is destination node, TSOD is that we search the paths with optimal condition started from node 1 and 3 respectively, meanwhile, after pass two paths, reach to their common destination which is the node 2. Also from the same source node to two different end node, referred to as a-source-two- destination (ASTD), as shown in Fig.2, in the roadway network diagram, bold path is corresponds to the optimal path, node 1 is the source node, node 2 and 3 is end node, ASTD is that we find the paths passed with optimal condition from node 1 to node 2 and 3 respectively. Another is that we can start from two different source nodes with optimal paths and reach to two different nodes, this is two-source-two-destination (TSTD), as shown in Fig.3, node 1 and 3 have two different source nodes, node 2 and 4 is two different end nodes, TSTD is that we search the paths passed with optimal condition from node 1 and 3 to node 2 and 4 respectively in the same tine. 


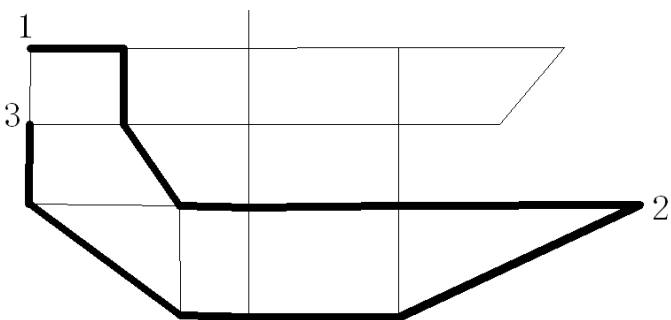

Fig.1 TSOD

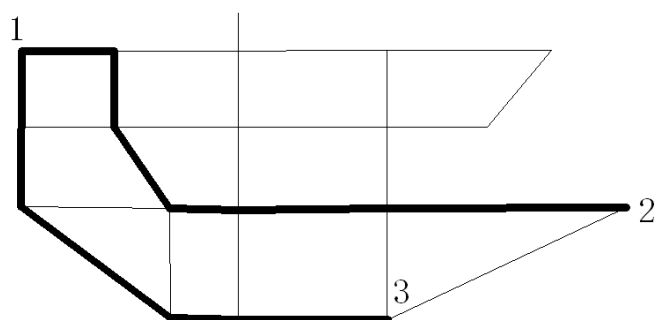

Fig.2 ASTD

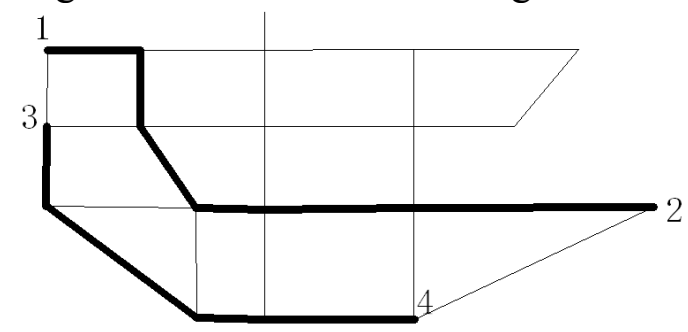

Fig.3 TSTD

In practice, the situation may be more complex, there is some factors that the optimal path analysis may be more than two source nodes and more than 2 termination nodes. No matter how much the source nodes and end nodes exist, the principle is the same algorithm, it is based on Dijkstra algorithm. The paper Improved Dijkstra algorithm that we used Binary heap priority queue to achieve Dijkstra algorithm.

Optimal path analysis of two sources has algorithm steps as follows:

Step1: Determine the type of optimal path analysis, namely we can choose one case from above three types of Optimal path analysis which are TSOD, ASTD and TSTD, then input source nodes and end nodes. If it exists mining levels, we should select the shortest path based on the hierarchical analysis; else if there is no level, we can directly choose improved Dijkstra algorithm based on binary heap.

Step2: In accordance with the optimal path type choose above to determine ID of source nodes(single or double) and destination nodes(single or double), meanwhile, we can select the method in terms of mine types to compute the optimal paths between one source node and one destination node. A set $(s, t)$ represents (one source node, one destination node). There are two set which are $(s, t)$ and $\left(s_{l}, t_{l}\right)$ need to be calculated, after computing, it will return paths sequence sets $\{s, \ldots, t\}$ and $\{s 1, \ldots, t 1\}$. If it is ASTD, then $s$ and $s l$ are the same node; else if it is TSOD, then $\mathrm{t}$ and $\mathrm{t} 1$ are the same node; else if it is TSTD, then $s$ and $s l$ are different nodes, $t$ and $t 1$ are different nodes.

Step3: combine all returned paths sequences by ordering.

The optimal path analysis with necessary nodes. In mine production and construction, we often encounter a situation where we start from a particular place within roadway, and must go through some locations(for example, water storage, avoid chamber, power distribution room ,etc.) which are the necessary nodes in roadway network, how to seek the optimal path pass through the these necessary nodes, not only to complete the task, also is the required cost most province, which is the problem exists the optimal path analysis need to be solved [12], as shown in Fig.4,

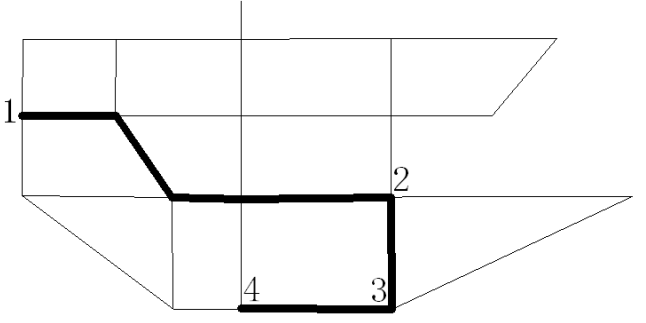

Fig.4 Necessary nodes 
Assume that the figure is a partial roadway network diagram, starting from the 1 st point, it must pass through the points sequence is $\{2,3,4\}$, but the final destination is the 4 th nodes. Under normal circumstances, the principle of optimal path analysis go through the necessary nodes is also used Dijkstra algorithm. Specific steps are as follows:

Step1:Input the source node $\mathrm{s}$, and node sequence set BJNodeSet $=\{a, b, \ldots, t\}$ which is must be passed through,

Step2: Take $s$ as a temporary source node, $a$ come from BJNodeSet is as a temporary termination node, in accordance with type of mine, we choose methods of path analysis to solve the optimal path between $s$ and $a$ node, it will return the path sequence $\operatorname{set}\{s, \ldots, a\}$.

Step3: Determine whether node $a$ is the termination node $t$, if it is, turn to step 5; if it is not, $a$ as temporary source node, the next node $b$ within BJNodeSet as temporary termination node, return step2 to solve the optimal path between $a$ and $b$, meantime, it will return the path sequence $\operatorname{set}\{a, \ldots, b\}$.

Step4: Judge whether node $b$ is the termination node $t$, if it is, turn to step 5; if it is not, turn to step 3 ;

Step5: The algorithm is completed; the paths sequences returned need to combine in order.

Take Fig. 4 for example, node 1 is source node, node 4 is termination node, and BJNodeSet is $\{2$, $3,4\}$, firstly, we should compute the node sequences of optimal path between node 1 and 2 , it will return $\{1, \ldots, 2\}$; then, judge whether node 2 is termination node $t$, node $2 \neq 4$, so, node 2 is not termination node, we must take node 2 as temporary source node, choose the next node 3 within BJNodeSet as temporary termination node. According to mine type, select the path analysis method to solve the optimal path from node 2 to node 3 , return the node sequence set of optimal path $\{2, \ldots, 3\}$, And so on, until the last node we choose coming from BJNodeSet is termination node, the algorithm exits, all branches of optimal paths have node sequences sets returned which need to be merge in, the whole optimal paths are obtained from node 1 to 4 , Bold line in Fig.4 is the optimal path between node 1 and 4.

The optimal path analysis with barriers. The optimal path analysis with barriers is also one of optimal path analysis, for example, in the network of roadway, there is a disaster which is drift gushing or roof collapse or fire appeared in a roadway induce to impassable, and this is an example that the barrier exists. The question is how to avoid the obstacles sections and go through to the destination. Similarly with the several types is that they are based on the Dijkstra algorithm which realized by using binary heap, so we can solve the optimal path analysis according to the types of mining. The specific algorithm steps of optimal path analysis with barriers is following:

Step1: Input the source node $s$ and destination node $t$.

Step2: according to the data structure of roadblocks, input barricades data which include roadblocks arcs name, node ID, weight and other roadblocks arcs.

Step3: replace the original path data with the data of roadblock arcs that is also setting the weight value of path to infinity.

Step4: In accordance with mine type to choose path analysis method, use the path data including replacement data for computing, return the path of optimal node sequence.

In Fig.5, in a local roadway network diagram, the dotted line represents that the roadway exists obstacles (gushing, roof collapse or fire, etc) and can not pass, that is to say node 2 and 3 is not connected (the weight value of arcs is set to infinity, the program is designed according to the specific circumstances, and here we set it to 99999), the node 7 and 8 is also not connected (the weight of the arc is also set to infinity). Like Node 1 as a source node, and node 6 as s termination node. If road section $(2,3)$ is connected, then the optimal path will pass through $(2,3)$, and arrive at node 6 which is the destination node, the thick lines in the figure represent that we take the roadblocks section into account in during of solving optimal path, namely roadway section $(2,3)$ can not be passed, so is roadway section $(7,8)$, at this time, the optimal path can only be the section from $(2,4)$ to $(4,5)$, and finally, reach to the destination node 6 . 


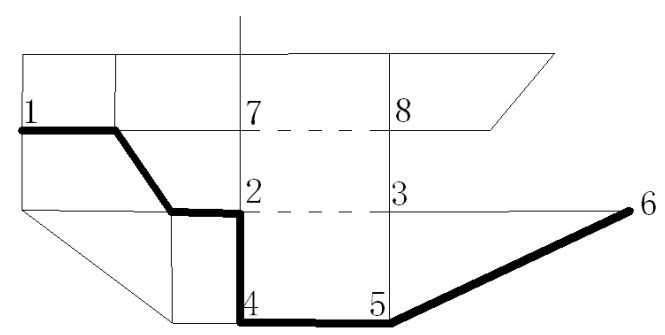

Fig.5 Barriers in path

\section{The analysis of experimental system}

Due to space limitations, this article will only take the optimal path analysis with barriers for example. In the paper, we take the roadway network of Huangling $1^{\text {st }}$ Mine for the research, and process the data of network firstly, Fig. 6 is a dialog about the optimal path analysis with necessary nodes, after reading the node file, arcs file and necessary nodes, the ID of source node and termination node are displayed apart, the summary nodes are known, node 3 is source node, the IDs of BJNodeSet are 19, 200 and 608, the termination node ID is 100 . Then, analyzing and calculating the path to obtain the arcs weights of optimal path, it is information included in the figure that there are 172 nodes and the optimal path length is nearly 9.3 kilometers. At the same time, all the paths' nodes are listed in order shown in the bottom of the list box, the nodes properties is shown and prepared by reference. Fig. 7 is shown the result of optimal path analysis with necessary nodes, and marked the corresponding node name, so that the optimal path analysis show results at a glance, there are necessary node 19(named 1A19) and 200(named E4) in Fig 7, node 100 is termination node.

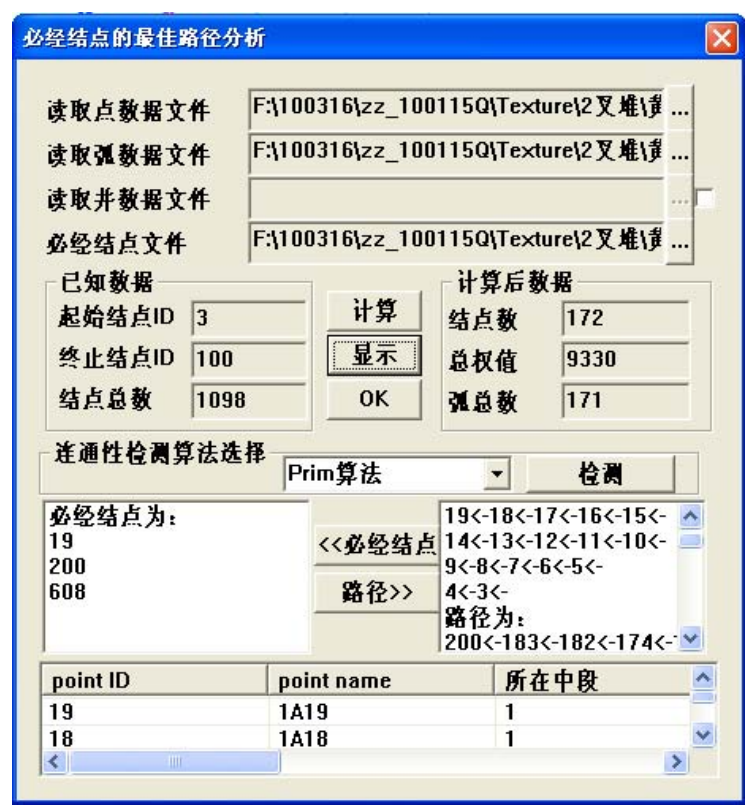

Fig. 6 The optimal path analysis with necessary nodes

Similarly, the analysis process is same among the multi-source optimal path analysis, optimal path analysis with barriers and optimal path analysis with necessary nodes, the principle used in the algorithm is same, just only choose different conditions.

According to above three optimal path analyses, it can assist mine roadway design, tunneling construction organization, decision-making and other disaster relief work. The study contributes to the development of digital mine theory, it is important for mine safety. In this paper, mine workings of specific geological factors, roadway maintenance and roadway usage are not considered, which will impact on the optimal path analysis when conducting optimal path analysis, which are the focus of this study for future research directions. 


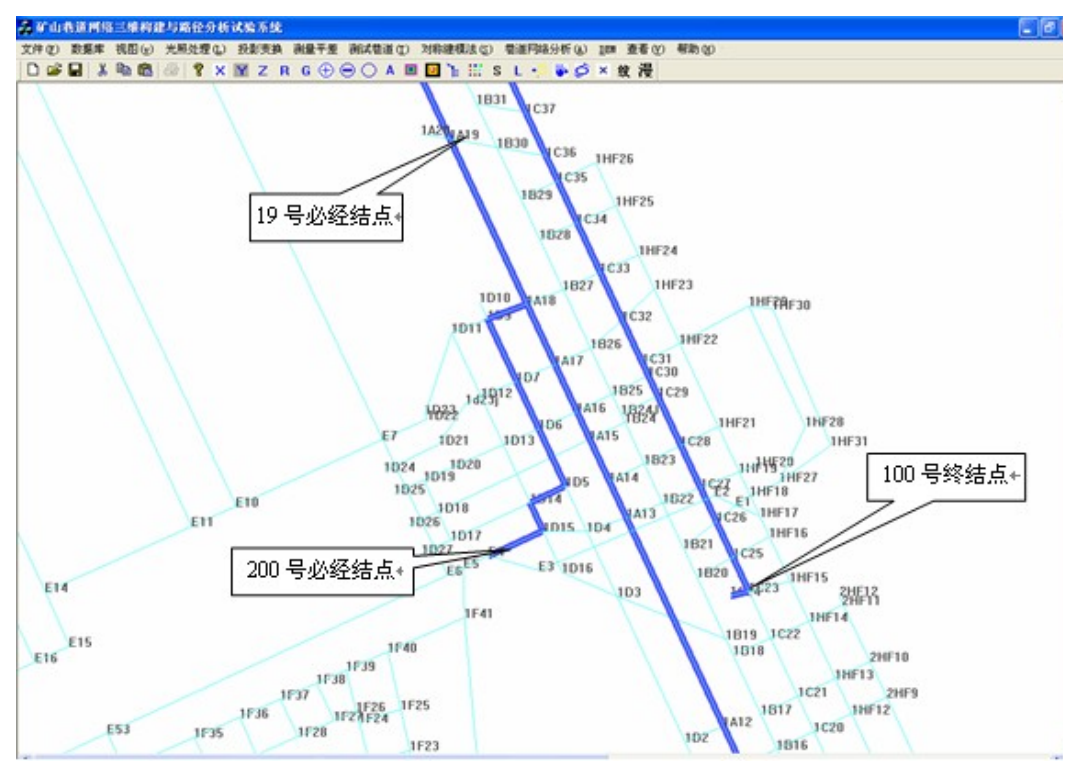

Fig. 7 Enlarged effect of optimal path analysis with necessary nodes

\section{Acknowledgment.}

The work was supported by a grant provided by National Natural Science Foundation of China (Project No. 41371435, 61462059), partially supported by a grant of the Jinchuan-Lanzhou Jiaotong University Fund, No. JCY2013015 and the Young Fund of Lanzhou Jiaotong University, No. 2015001.The authors are grateful to the editors and anonymous reviewers more enough for their self-giving works.

\section{References}

[1] Y.Q. Shao Y.J. Wang. Establishment and Analysis of Roadway-oriented Geometric Network Model. Mining Research \& Development, Vol.31 (2011), p. 56-59

[2] Z.H. Zhang, E.K. Hou, Z. Zhao, et al. Application of GIS Network Analysis in Mine. Geomatics World. Vol.6 (2008), p. 39-43

[3] S. Xia Y.S. Han. An Improved Implementation of Shortest Path Algorithm in GIS. Bulletin of surveying and mapping. (2004), p. 40-42

[4] N.N. Li, Y.S. Liu. Application of Modified Dijkstra Algorithm in GIS Route Planning. Computer and Modernization. (2004), p. 12-17

[5] W.W. Tang, X.D. Shi,D.K. Zhu. The Calculation of the Shortest Path Using Modified Dijkstra Algorithm in GIS. Journal of Image and Graphics. Vol.5 (2000), p. 1019-1023.

[6] Y. Yue J.Y. Gong. An Efficient Implementation of Shortest Path Algorithm Based on Dijkstra Algorithm. Journal of Wuhan Technical University of Surveying and Mapping. Vol.24 (2011), p. 209-212.

[7] F. Lu, D.M. Lu and WH. Cui. Improved Dijkstra Algorithm Based on Quad-Heap Priority Queue and Inverse Adjacent List[].Journal of Image and Graphics. Vol.4 (2011), p. 1039-1045.

[8] H.Zhang, Y.N Wen, A.L Liu, , et al. Foundation Algorithm of Geographic Information System, China: Beijing:Science Press(2006).

[9] L. X. Wu, W. Z. Shi. Principles and Algorithms of GIS, China: Beijing:Science Press(2003).

[10] W. Chen, T.R. Yao, J.X. Hong. Research on an Isochrone Approach and Its Shortest Routing Algorithms Based on GIS. Journal of Wuhan University of Technology. Vol.26 (2002), p. 466-469. 
[11] Z.H. Zhang: Research on Method of 3D Tunnel Network Modeling and Path Analysis. Ph.D, Xi'an: Xian University of Science and Technology (2010). 